\title{
COMPARACIÓN PRODUCTIVA DE FORRAJE VERDE HIDROPÓNICO DE MAÍZ, ARROZ Y SORGO NEGRO FORRAJERO ${ }^{1}$
}

\author{
Claudio Fabián Vargas-Rodríguez ${ }^{2}$
}

\begin{abstract}
RESUMEN
Comparación productiva de forraje verde hidropónico de maíz, arroz y sorgo negro forrajero. La investigación se desarrolló en la Estación Experimental "Alfredo Volio Mata" de la Universidad de Costa Rica durante el mes de agosto del año 2006, donde se evaluaron tres forrajes (Zea mays, Sorghum almun y Oryza sativa) bajo un sistema de producción hidropónico. La mayor producción de biomasa fresca $\left(21,65 \mathrm{~kg} / 720 \mathrm{~cm}^{2}\right)$ fue dada por el sorgo que a su vez manifestó una mayor concentración de proteína cruda (10,47\%). El arroz resultó ser la especie con contenidos de materia seca $(15,82 \%)$ y cenizas $(9,17 \%)$ superior, mientras que el maíz presentó una mejor calidad de fibra. Con estos resultados se puede deducir que los sistemas de producción hidropónica representan una alternativa más para el cultivo rápido y simple de forraje durante las épocas adversas.
\end{abstract}

Palabras clave: Forraje, hidroponía, nutrición animal, pastos, cultivos.

\begin{abstract}
Comparison of production yields of green hydroponic fodder of corn, rice and sorghum. The investigation was developed at Experimental Station "Alfredo Volio Mata" of the University of Costa Rica during August, 2006. Three materials (Zea mays, Sorghum almun and Oryza sativa) were evaluated under hydroponic production systems. The highest production of fresh matter was given by Sorghum $(21,65$ $\mathrm{kg} / 720 \mathrm{~cm}^{2}$ ) that also showed the highest concentration of Crude Protein $(10,47 \%)$. Rice was the material with superior levels of dry matters $(15,82 \%)$ and ash $(9,17 \%)$, while corn showed the fibber with highest quality. These results indicate that production of forage under hydroponics systems represents an alternative to grow forage fast and easy during adverse periods.
\end{abstract}

Keywords: Forage, hydroponics, animal nutrition, pasture, crops.

\section{INTRODUCCIÓN}

Los sistemas de producción bovina sustentan sus prácticas alimenticias en el componente forrajero, elemento que es considerado como el insumo de menor costo a través del cual es posible suplir gran parte de las demandas nutricionales de los animales en producción (Fumagalli y Kunst 2002).

Existe una interdependencia entre el suelo como medio de soporte radical del cultivo, la pastura como fuente de alimentación y el componente animal, factores que conjugados determinan la complejidad de los sistemas de explotación ganaderos, y requieren de tiempos prolongados para comprobar la respuesta a cualquier cambio que permita adecuar la oferta forrajera de acuerdo a las demandas de la explotación (De León 2004).

No obstante los sistemas de producción de forraje convencional han venido experimentando serias dificultades marcadas por la situación actual del sector agropecuario, el intenso crecimiento en la tasa de urbanización

\footnotetext{
Recibido: 29 de agosto, 2007. Aceptado: 26 de agosto, 2008. Trabajo financiado por la Vicerrectoría de Investigación. Proyecto VI-737-A4-133.

2 Estación Experimental Alfredo Volio Mata, Facultad de Ciencias Agroalimentarias, Universidad de Costa Rica, Cartago, Costa Rica. fabian. vargas@ucr.ac.cr
} 
y el aumento en el valor de las tierras centrales se han encargado de desplazar las explotaciones pecuarias hacia sectores donde se reduce el potencial de producción forrajero (Fernández, citado por Pezo et al. 1996).

Aunado a lo antes mencionado, la necesidad de intensificar y mejorar la eficiencia en las prácticas de producción animal de una manera sostenible, el incremento en la demanda de productos alimenticios, la expansión de la frontera agrícola y ganadera, la erosión del suelo y la contaminación de las aguas, el crecimiento estacional de los pastos debido a la estacionalidad de las lluvias, son algunos de los factores que han dirigido la investigación hacia la búsqueda de métodos alternos de producción de alimentos (Money 2005, Rotar 2006).

Como una alternativa importante, se gesta la producción de forraje verde hidropónico, que se trata de una tecnología de producción de biomasa obtenida a partir del crecimiento inicial de las plantas en los estados de germinación y crecimiento temprano de plántulas a partir de semillas viables (FAO 2001) .

La hidroponía se basa en la producción de plantas en soluciones nutritivas líquidas en lugar de utilizar el suelo como sustrato. La mayoría de los trabajos han centrado su aplicación en vegetales y hortalizas, no obstante orientado hacia la producción de alimento para ganado y otras especies animales generando un producto altamente nutritivo, rico en enzimas y vitaminas que se pueden desarrollar a escalas industriales que aumentarían el rendimiento por área (Rotar 2004).

El forraje hidropónico es el resultado del proceso de germinación de granos que se realiza durante un periodo de 9 a 15 días. Pretendiendo que el grano germinado alcance una altura promedio de 25 centímetros (Chang et al. 2002). No obstante Henriques, citado por Müller et al. (2005) menciona que una edad de cosecha adecuada del cultivo puede estar entre 16 y 20 días de acuerdo a las necesidades del productor, sin pasar ese periodo de tiempo. Durante el proceso de germinación de una semilla se producen una serie de cambios que le permiten a la plántula en pocos días captar energía luminosa y a través de un proceso de crecimiento acelerado desarrollar su parte radicular y aérea con muy poco contenido de fibra y altos contenidos de aminoácidos en forma libre y que se aprovechan fácilmente por los animales (Valdivia 1997).

Con el forraje hidropónico se puede alimentar ganado vacuno, porcino, caprino, equino, cunícola y una gran cantidad de animales domésticos con excelentes resultados. Entre las ventajas que presenta el forraje hidropónico, se puede decir que: permite un suministro constante durante todo el año, se pueden emplear terrenos marginales, se reduce el desperdicio de agua, se obtiene una fuente alternativa de alto valor nutricional, es completamente natural por lo que hay una menor incidencia de enfermedades, se puede dar un aumento en la fertilidad y la producción de leche (Money 2005). En general, todas las ventajas que los animales puedan obtener de una buena alimentación.

El objetivo del presente trabajo fue evaluar el rendimiento productivo y contenido de nutrimentos de tres especies de gramíneas con potencial forrajero: maíz criollo (Zea mays), arroz (Oriza sativa) y sorgo negro forrajero (Sorghum almun) bajo un sistema de cultivo.

\section{MATERIALES Y MÉTODOS}

\section{Localización}

El trabajo de investigación se realizó durante el mes de agosto del año 2006 en la Estación Experimental de Ganado Lechero Alfredo Volio Mata de la Universidad de Costa Rica, localizada a $1542 \mathrm{msnm}$, en Ochomogo de Cartago; presenta una temperatura media de $19,5{ }^{\circ} \mathrm{C}$ con una precipitación promedio de $2.050 \mathrm{~mm}$ anuales.

\section{Materiales experimentales}

Se evaluaron tres gramíneas con potencial para producción forrajera: maíz criollo (Zea mays) código VAR LD-8843, arroz (Oriza sativa) código CR-4477 y sorgo negro forrajero (Sorghum almun) código UCREEAVM, bajo un sistema de cultivo hidropónico. Para tal efecto se implementó para los tres materiales el procedimiento de producción citado por Valdivia (1997), Carballo (2000), Chang et al. (2002), Carballido (2005) y Elizondo (2005). 


\section{Procedimiento de cultivo}

1. Pesaje y escogencia de las semillas: Se utilizó la recomendación de acuerdo a Carballo (2000) en la cual indica $1,7 \mathrm{~kg}$ para bandejas de $0,269 \mathrm{~cm}^{2}$ por lo que se utilizó la proporción de $4 \mathrm{~kg}$ para bandejas de $720 \mathrm{~cm}^{2}$. Se hizo una selección manual de las semillas para eliminar todas aquellas que estaban en mal estado (semillas partidas) y cuerpos extraños.

2. Prelavado: En baldes de remojo se colocó por separado cada grupo de las semillas y de las diferentes gramíneas a evaluar, y se eliminaron todas aquellas que flotaron en agua.

3. Lavado: Las semillas se lavaron y desinfectaron en una solución de hipoclorito de sodio $(5,25 \%)$ al $1 \%$ (10 ml de solución de cloro comercial en un litro de agua) dejándolas remojar en ésta por 30 minutos a una hora, luego se enjuagaron con agua.

4. Remojo: Se sumergieron las semillas en agua por un periodo de tiempo de 18-20 horas, se hizo recambio manual del agua que se veía turbia.

5. Oreo: Las semillas se colocaron sobre sacos limpios extendidos para orearlos por un lapso de 24 horas.

6. Traslado: Se colocaron las semillas en las bandejas de un tamaño de $720 \mathrm{~cm}^{2}$ tratando de formar una capa uniforme de $1,5 \mathrm{~cm}$ de espesor, se utilizaron cuatro bandejas por gramínea.

7. Germinación: Para lograr una adecuada germinación, las bandejas fueron cubiertas con un plástico negro (Figura 1), y se colocaron en un invernadero donde se mantuvo a baja temperatura, buena ventilación, oscuridad y con la ayuda de nebulizadores de riego se suministró el agua evitando que se movieran las semillas, este proceso duró 24 horas.

8. Producción: Una vez pasado el tiempo de germinación, se retiró el plástico negro que cubrió las bandejas, en este momento se iniciaron los riegos espaciados cada tres horas por 30 segundos, para tales efectos se diseñó un sistema de suministro de agua por aspersión equipado con nebulizadores y un dosificador de nutrientes (Figura 2).

Al aparecer las primeras hojas se comenzó la etapa de fertirrigación (aproximadamente al cuarto día) utilizando una combinación de soluciones nutritivas descritas en los Cuadros 1 y 2 (5 ml de A y 2,5 $\mathrm{ml}$ de B) hasta el sétimo día, luego se procedió a regar con agua solamente. En este lapso de tiempo se le suministró a los cultivos luz, temperatura adecuada, buena ventilación y cuidados sanitarios. Esta parte duró 10 días momento en el cual los cultivos alcanzaron la altura de $25 \mathrm{~cm}$ de altura.

9. Cosecha: Se realizó cuando las tres especies evaluadas alcanzaron $\operatorname{los} 25 \mathrm{~cm}$ de altura.

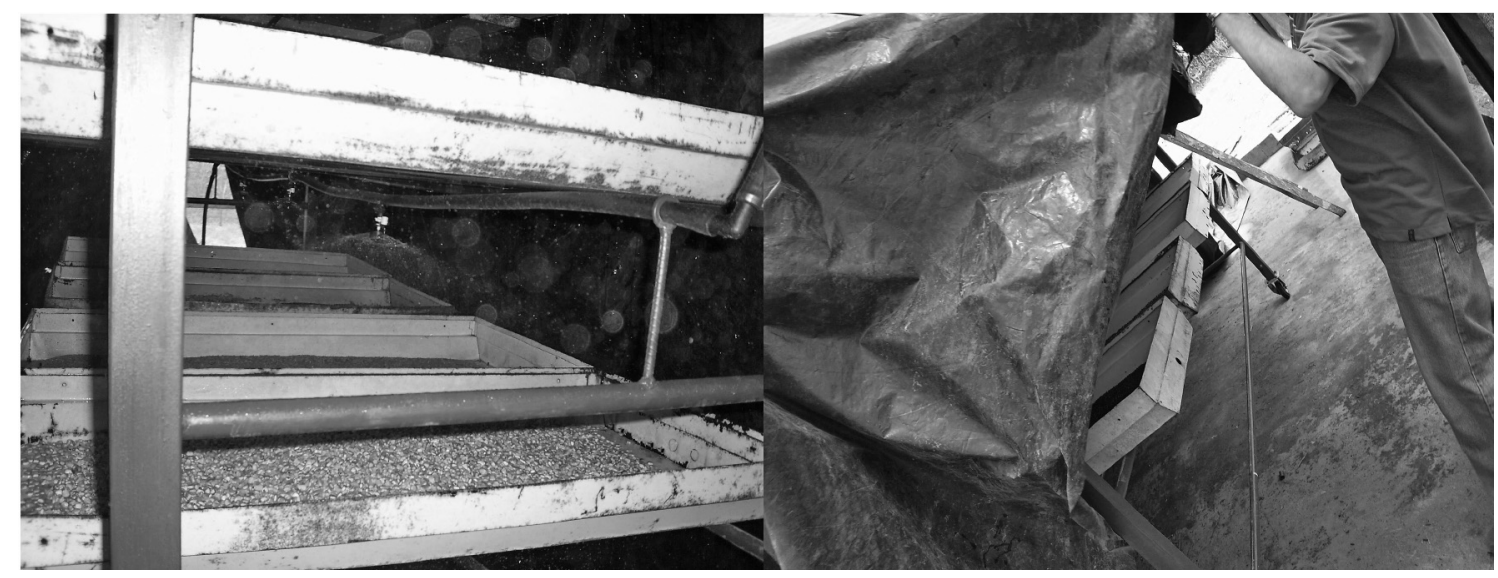

Figura 1. Control de luz durante el proceso de germinación de semillas de maíz, arroz y sorgo negro, para forraje verde hidropónico. Cartago, Costa Rica. 2007. 


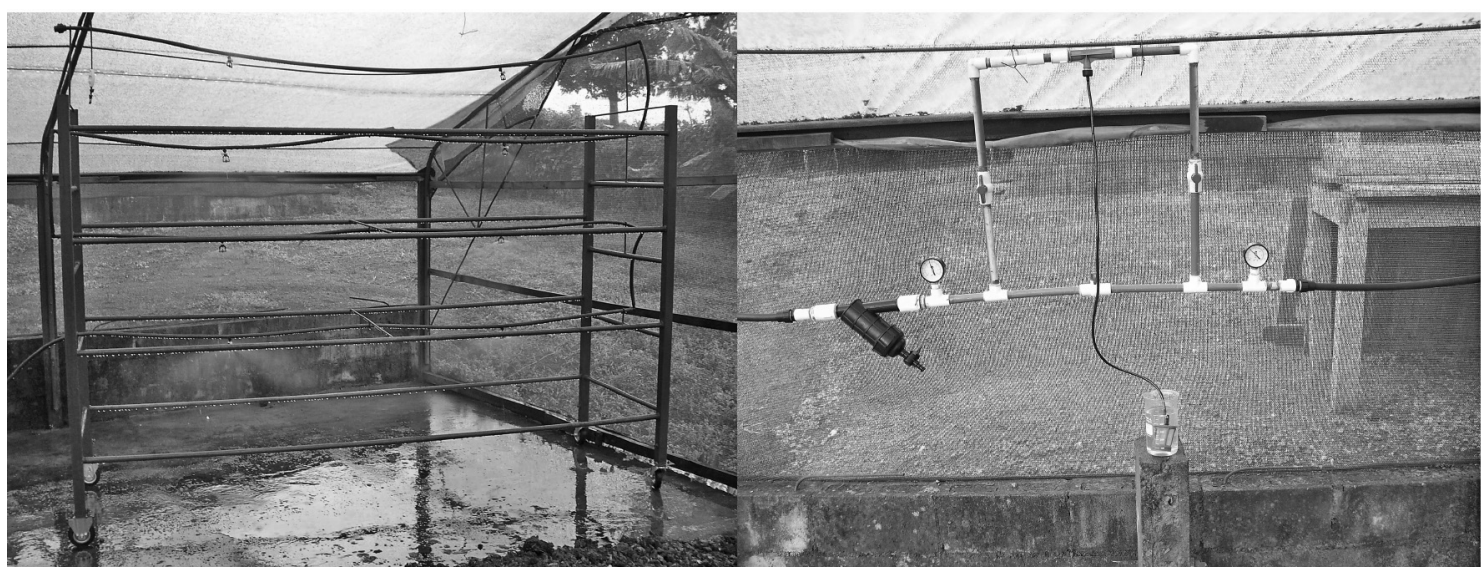

Figura 2. Estantería para producción de forraje verde hidropónico con el equipo de riego y el dosificador de nutrientes. Cartago, Costa Rica. 2007.

Cuadro 1. Detalle de la solución nutritiva concentrada en elementos mayores utilizada durante el periodo de fertirrigación. Cartago, Costa Rica. 2007.

\begin{tabular}{lccc}
\hline $\begin{array}{c}\text { Dosificación 5 ml/l } \\
\text { Solución A }\end{array}$ & \multicolumn{2}{c}{ Composición } & Concentración \\
\hline Nitrógeno & $5 \%$ & $5,00 \%$ de $\mathrm{N}$ & $250 \mathrm{ppm}$ \\
Fósforo & $2 \%$ & $4,60 \%{\mathrm{de} \mathrm{P}_{2} \mathrm{O}_{5}}$ & $100 \mathrm{ppm}$ \\
Potasio & $4 \%$ & $4,81 \% \mathrm{de} \mathrm{K}_{2} \mathrm{O}$ & $200 \mathrm{ppm}$ \\
Calcio & $2 \%$ & $2,80 \%$ de CaO & $38 \mathrm{ppm}$ \\
Magnesio & $0,75 \%$ & $1,30 \%$ de $\mathrm{MgO}$ & $100 \mathrm{ppm}$ \\
\hline
\end{tabular}

Cuadro 2. Detalle de la solución nutritiva concentrada en elementos menores utilizada durante el periodo de fertirrigación. Cartago, Costa Rica 2007.

\begin{tabular}{lcc}
\hline $\begin{array}{l}\text { Dosificación 2,5 ml/l } \\
\text { Solución B }\end{array}$ & Composición & Concentración \\
\hline Magnesio & $28.000 \mathrm{ppm}$ & $70,0 \mathrm{ppm}$ \\
Azufre & $20.180 \mathrm{ppm}$ & $70,0 \mathrm{ppm}$ \\
Hierro & $1.560 \mathrm{ppm}$ & $3,9 \mathrm{ppm}$ \\
Cobre & $100 \mathrm{ppm}$ & $0,2 \mathrm{ppm}$ \\
Boro & $375 \mathrm{ppm}$ & $0,9 \mathrm{ppm}$ \\
Manganeso & $400 \mathrm{ppm}$ & $1,0 \mathrm{ppm}$ \\
Zinc & $252 \mathrm{ppm}$ & $0,6 \mathrm{ppm}$ \\
Molibdeno & $24 \mathrm{ppm}$ & $0,006 \mathrm{ppm}$ \\
Sodio & $215 \mathrm{ppm}$ & $0,5 \mathrm{ppm}$ \\
Cloro & $220 \mathrm{ppm}$ & $0,5 \mathrm{ppm}$ \\
\hline
\end{tabular}

\section{Estimación de biomasa}

Para estimar la producción de biomasa, se cosechó el material cuando el cultivo alcanzó la edad de 20 días, y como criterio de cosecha se tomó en consideración la altura del cultivo $(25 \mathrm{~cm})$ (Henriques, citado por Müller et al. 2005, Carballido 2005, Elizondo 2005), el material fresco se pesó de inmediato y se procedió a extraer cinco muestras representativa de $2 \mathrm{~kg}$ incluyendo raíces y semillas, luego fue ubicado en una estufa a $60^{\circ} \mathrm{C}$ hasta lograr un peso constante. Posteriormente para los análisis de laboratorio correspondientes se molió utilizando un molino marca Wiley con tamiz de $1 \mathrm{~mm}$.

Como modelo experimental se empleó un diseño irrestrictamente al azar y los datos fueron analizados con el PROC ANOVA de SAS, versión 8.2 (SAS 1999). La prueba de medias se efectuó mediante una comparación de DUNCAN.

\section{Análisis de laboratorio}

La materia seca (MS) total se obtuvo a partir de una muestra sometida a una temperatura de $105^{\circ} \mathrm{C}$, y luego se determinó el contenido de cenizas mediante una combustión a $550{ }^{\circ} \mathrm{C}$. La fibra neutro detergente (FND), la fibra ácido detergente (FAD) y la lignina se determinaron mediante el método de Goering y Van Soest (1970), y la celulosa y hemicelulosa, fueron 
estimadas por diferencia. El contenido de proteína cruda (PC) se evaluó por el método de Kjeldahl (Sosa de Pro 1979).

\section{RESULTADOS Y DISCUSIÓN}

El mayor rendimiento de biomasa se obtuvo en sorgo negro forrajero. En promedio, las bandejas de $720 \mathrm{~cm}^{2}$ de esta semilla lograron producir $21,65 \mathrm{~kg}$ de forraje verde hidropónico $(\mathrm{FVH})$. Por su parte, el maíz alcanzó el segundo nivel de producción generando $17,20 \mathrm{~kg}$ de biomasa y por último el arroz, cuyos rendimientos no superaron los 14,35 kg. Entre la producción del sorgo y la de maíz no hubo diferencias significativas $(\mathrm{P}>0,05)$, igual sucedió entre el maíz y el arroz; entre el sorgo y el arroz las diferencias fueron significativas al $8 \%$.

La relación semilla: material producido en el caso del sorgo fue de 1: 5,45, para el maíz de 1: 4,3 y para el arroz de 1: 3,58. De acuerdo con Tarrillo (2007) a partir de $1 \mathrm{~kg}$ de semilla se puede producir una masa forrajera de 6 a $8 \mathrm{~kg}$ consumible en su totalidad. Por su parte, Elizondo (2005) menciona que a partir de 1 $\mathrm{kg}$ de semilla se pueden obtener $9 \mathrm{~kg}$ de biomasa; $\sin$ embargo, en el presente trabajo no se logró alcanzar esos rendimientos; no obstante, coincide con lo establecido por Valdivia (1997) y Sneath y McIntosh (2003) quienes indican que se puede considerar un buen rendimiento en forrajes bajo sistemas hidropónicos cuando la relación se mantiene en 1: 5 , además Valdivia (1997) menciona que obtener mayor volumen de material resulta complicado debido a la limitante, en este caso, de la calidad de la semilla disponible.
Como se observa en el Cuadro 3 la gramínea con el porcentaje de MS más alto fue el arroz que mostró diferencias altamente significativas $(\mathrm{P}<0,001)$ con respecto al maíz y el sorgo, estos últimos entre sí no resultaron diferentes. Estos resultados concuerdan con lo mencionado por Tarrillo (2007) quien recopilando información de varios autores aduce que es posible obtener valores de MS entre 12-20\% en sistemas hidropónicos; esto se asemeja a lo encontrado por Espinoza et al. (2004) quienes en un estudio utilizando forraje verde hidropónico $(\mathrm{FVH})$ para alimentar toretes mestizos obtuvieron maíz con un 14,43\% MS, y también concuerda con Rodríguez (2000) quien menciona que dependiendo de la especie forrajera es posible obtener materiales que varían entre 12 y $18 \%$ MS. No obstante, los resultados no coinciden con los valores mencionados por otros autores quienes aseveran que los rendimientos esperados en cuanto a $\%$ MS rondan entre 20 y 30\% (Carballo 2000, FAO 2001, Elizondo 2005, Müller et al. 2005).

El contenido de nitrógeno en los materiales hidropónicos es mayor a edades tempranas (10 días). Lo anterior se debe a que en las plantas jóvenes el crecimiento está relacionado principalmente, con un aumento en la superficie de las hojas que son los órganos ricos en nitrógeno (Müller et al. 2005). Con un incremento en la edad, las partes estructurales y de acumulación como tallo y pecíolos, que son más pobres en nitrógeno, se tornan preponderantes, así mismo las necesidades de ese elemento para la síntesis de biomasa son menores. Bajo esas condiciones el nitrógeno de las hojas es removido para las partes jóvenes, la fracción de biomasa activa disminuye lo que acentúa la dilución del nitrógeno en la planta (Andriolo 1999, Taiz y Zerge 2004).

Cuadro 3. Composición bromatológica de las especies cosechadas bajo el sistema de forraje verde hidropónico. Cartago, Costa Rica 2007.

\begin{tabular}{lcccccccc}
\hline & \multicolumn{7}{c}{ CONTENIDOS PORCENTUALES } \\
\cline { 2 - 8 } Material & MS & PC & Cenizas & FND & Celulosa & Hemiceluosa & Lignina & FAD \\
\hline Arroz & $15,82^{\mathrm{a}}$ & $7,92^{\mathrm{a}}$ & $9,17^{\mathrm{a}}$ & $58,25^{\mathrm{a}}$ & $27,76^{\mathrm{a}}$ & $19,82^{\mathrm{a}}$ & $10,67^{\mathrm{ab}}$ & $38,54^{\mathrm{a}}$ \\
Maíz & $11,54^{\mathrm{b}}$ & $9,61^{\mathrm{b}}$ & $2,41^{\mathrm{b}}$ & $43,13^{\mathrm{b}}$ & $11,21^{\mathrm{b}}$ & $24,25^{\mathrm{a}}$ & $7,67^{\mathrm{b}}$ & $18,89^{\mathrm{b}}$ \\
Sorgo & $11,48^{\mathrm{b}}$ & $10,47^{\mathrm{c}}$ & $6,54^{\mathrm{c}}$ & $66,66^{\mathrm{c}}$ & $30,96^{\mathrm{a}}$ & $21,42^{\mathrm{a}}$ & $14,28^{\mathrm{a}^{\mathrm{a}}}$ & $45,17^{\mathrm{c}}$ \\
\hline
\end{tabular}

Letras diferentes entre filas muestran diferencias significativas $(\mathrm{P}<0,01)$ 
$\mathrm{Al}$ analizar el contenido de $\mathrm{PC}$ presente en los materiales en estudio, el sorgo se manifestó como el forraje con mejores condiciones. Presentó 10,47\% de ese nutriente, $\mathrm{y}$ las diferencias con respecto a otras especies evaluadas fueron altamente significativas $(\mathrm{P}<0,001)$. El arroz reflejó ser el punto opuesto con un contenido de $7,92 \%$ mientras que el maíz produjo un $9,61 \%$.

Es posible que los bajos contenidos de PC se deban a la edad de cosecha que se implementó en este trabajo (20 días). Sandia, citado por Müller et al. (2005) menciona que pueden haber reducciones conforme avanza la madurez del cultivo, pues aduce que a una edad de 12 días obtuvo FVH de maíz con 17,4\% de PC y a los 14 días se redujo a 13,4\%; Balerio et al. (2000) complementan esta afirmación, cuando trabajando con maíz hidropónico obtuvieron valores de $11,7 \%$ a los 16 días. Por su parte, Carballido (2005) cosechando a edades de 12 días, obtuvo 18,80\% para FVH de maíz, $25,75 \%$ para cebada cervecera y $19,4 \%$ para cebada blanca y por Espinoza et al. (2004) quien cosechó un maíz hidropónico que promedió 19,44\% PC. Sin embargo, el contenido mínimo de proteína cruda que debe tener un FVH es de $7 \%$ que si se logra en el presente trabajo, lo que garantiza que se pueda dar como mínimo la fermentación de los carbohidratos estructurales a nivel de rumen (Van Soest 1994), aunque Tarrillo (2007) considera que rangos normales en producción hidropónica deben variar entre 12-25\% PC.

El contenido de cenizas permitió conocer la concentración mineral de los cultivos, en el caso del arroz fue de $9,17 \%$, para el sorgo y maíz fueron respectivamente $6,54 \%$ y $2,41 \%$. El análisis estadístico demostró diferencias altamente significativas entre ellos $(\mathrm{P}<0,01)$; los niveles que mostraron el arroz y el sorgo fueron muy altos, posiblemente eso se debe a la cutícula que recubre la semilla de ambos materiales con valores de $2 \%$ y $1,84 \%$ obtenidos respectivamente por FAO (2001) y Carballo (2000), caso contrario que sucede con el maíz.

La composición de la pared celular define el contenido fibroso de los materiales utilizados para la alimentación de rumiantes y está integrada por celulosa, hemicelulosa, lignina, pectina y otros componentes, su importancia se centra en promover la producción de ácidos grasos volátiles que al final se traducen en aumentos del contenido graso en la leche. Espinoza et al. (2004) encontraron que los niveles de FND para maíz en FVH eran cercanos a 41,46\%, mientras que Müller et al. (2002) obtuvieron valores de $80 \%$ y $64,9 \%$ de FND para maíz producido hidropónicamente bajo diferentes densidades de siembra.

Los resultados obtenidos en el presente estudio concuerdan con los autores mencionados en el párrafo anterior, dado que el contenido de FND obtenido en el sorgo fue de 66,59\%; mientras que en el arroz fue 58,36\% y el maíz alcanzó apenas un 43,15\%. El análisis estadístico respaldó las diferencias entre los tres materiales, demostrando que éstas eran altamente significativas $(\mathrm{P}<0,001)$. De acuerdo con NRC (2001) las dietas de vacas durante su periodo de lactación debe contener como mínimo 25\% de FND de la cual el 75\% lo suple el forraje de manera que se asegura la producción de saliva suficiente para regular el pH ruminal.

Si se separan las fracciones de la fibra (Cuadro 3), es apreciable que el contenido de carbohidratos aprovechables (hemicelulosa y celulosa) por los microorganismos del rumen, son muy parecidos entre el arroz y el sorgo, la celulosa y hemicelulosa no presentaron diferencias significativas $(P>0,05)$ entre ellos, mientras que el maíz resultó ser diferente significativamente $(\mathrm{P}<0,05)$ a ambos pero solo en la porción celulolítica.

Por otra parte al hacer la extracción con la solución ácido detergente, la tendencia fue muy parecida a la FND, donde el análisis estadístico justificó que el sorgo resultó ser significativamente $(\mathrm{P}<0,05)$ superior al arroz y al maíz $(\mathrm{P}<0,001)$. Los valores obtenidos del maíz $(18,89 \%)$ se asemejan más a lo expuesto por Espinoza et al. (2004) quien obtuvo 20,94\% de FAD en maíz hidropónico y son comparables con Sneath y McIntosh (2003) y con FAO (2001) quienes mencionan rangos desde $18 \%$ hasta $28,2 \%$ pero en cebada y avena. Por otra parte, los niveles obtenidos en sorgo y arroz se asemejan a los datos de $56,7 \%, 49,7 \%$ y $42,5 \%$ de FAD obtenidos por Isepon et al. (2005) cuando trabajó un cultivo hidropónico de maíz a diferentes edades de siembra.

A partir de aquí se determinó el porcentaje de lignina que presentaron estos materiales, donde el maíz mostró el nivel más bajo y por ende la calidad de su fibra resultó mayor, no obstante no mostró diferencias $(\mathrm{P}<0,05)$ con respecto al arroz, en el cual se esperarían 
valores más altos debido al recubrimiento que tiene su semilla el cual se mantiene aprisionado entre las raíces desarrolladas, situación que no sucedió. Sin embargo, el sorgo tuvo un valor muy alto en este elemento y por ende puede ser considerado el material más limitante para ser utilizado en sistemas hidropónicos a pesar de contener el nivel de PC más alto.

\section{CONCLUSIONES}

El sorgo fue la especie que rindió más en biomasa fresca, incluso fue el de mayor producción de PC, no obstante su nivel de fibra podría inducir a una reducción en el consumo y en el aprovechamiento del mismo.

El maíz resultó ser el material más suculento por sus niveles de fibra, complementado con un buen nivel de PC. No obstante su punto alto podría ser la concentración de energía que puede aportar a esta edad.

El arroz se manifestó como una buena fuente de minerales pero con un bajo nivel de proteína, además su rendimiento de biomasa fue muy bajo a pesar de que su contenido porcentual de MS fuera el más alto.

Los materiales hidropónicos pueden considerarse una buena fuente de minerales para la alimentación animal, sobre todo el arroz y el sorgo.

Es necesario enfocar más trabajos en producción de forraje hidropónico e implementar más variedad de cultivos, dado que la mayoría de las investigaciones se centran sobre el maíz, y es importante brindarle más opciones a los productores.

El factor más limitante de la producción de FVH es la calidad de la semilla y la dificultad de conseguirla.

\section{LITERATURA CITADA}

Andriolo, J. 1999. Fisiología das culturas protegidas. Santa María, Brasil. Editorial UFSM. p. 142.

Balerio, G; Ferreira, A; Viana, M; Resende, E; Cruz, C. 2000. Produção de forragen hidropônica de milho con diferentes substratos. Brasil. Anuario $37^{\circ}$. Reunión

ISSN: 1021-7444
Anual de la Sociedad Brasileña de Zootecnia. Viçosa: SBZ, CD ROM. Forragicultura.

Carballido, C. 2005. Forraje verde hidropónico. Artículos silvoagropecuarios: Forraje verde hidropónico (en línea). Chile. Consultado el 15 jun. 2007. Disponible en: http://www.ofertasagricolas.cl/articulos/88

Carballo, C. 2000. Manual de procedimientos para germinar granos para la alimentación animal (en línea). Culiacán, México. Consultado 22 jun. 2007. Disponible en: http://www.zoetecnocampo.com/Documentos/germinados.htm

De León, M. 2004. Herramientas para manejar las complejas relaciones "pastura-animal". Córdoba, Argentina. Producción de Carne Bovina. Boletín Técnico Producción Animal 2 (1).

Elizondo, J. 2005. Forraje verde hidropónico. Una alternativa para la alimentación animal. Revista ECAG informa (32): 36-39

Espinoza, F; Argeti, P; Urdaneta, G; Areque, C; Fuentes, A; Palma, J; Bello, C. 2004. Uso del forraje del maíz (Zea mays) hidropónico en la alimentación de toretes mestizos. Venezuela. Revista Zootecnia Tropical 22(4): 303-315.

FAO (Organización de las Naciones Unidas para la Agricultura y la Alimentación, IT). 2001. Manual técnico: Forraje verde hidropónico. Oficina Regional de la FAO para América Latina. Santiago, Chile. 68 p.

Fumagalli, A; Kunts, C. 2002. Cómo mejorar la oferta forrajera de los sistemas de cría. Cadena de la Carne Vacuna. Tecnologías para nuevos escenarios. Revista IDIA XXI: N² 2002. p. 73-78.

Goering, HK; Van Soest, PJ. 1970 Forage fiber analysis (apparatus, reagents, procedures and some applications). Agricultural Handbook No. ARS-USDA, Washington, DC. 76 p.

Isepon, O; Silva, A; Matsumoto, E; Campos, Z. 2005. Zootecnia Tropical 23(2): 105-119.

Mooney, J. 2005. Growing cattle feed hydroponically. 2002 Scholarship Report. Australian Nutffield Farming Scholars Association. Australia. 30 p.

AGRONOMÍA MESOAMERICANA 19(2): 233-240. 2008 
Müller, L; Manfron, P; Santos, O; Medeiros, S; Haut, V; Dourado, D; Binotto, E; Bandeira, A. 2005. Producción y composición bromatológica de forraje hidropónico de maíz (Zea mays L.) con diferentes densidades de siembra y días de cosecha. Brasil. Zootecnia Tropical 23(2): 105-119.

NRC (National Research Council). 2001. Nutrient requirements of dairy cattle. 7 ed. Washington D.C. National Academy of Science -National Research Council. $381 \mathrm{p}$.

Pezo, D; Holfmann, F; Arze, J. 1996. Evaluación bioeconómica de un sistema de producción de leche basado en el uso intensivo de gramíneas fertilizadas en el trópico húmedo de Costa Rica. Agronomía Costarricense 23 (1): $105-117$.

Rodríguez, S. 2000. Hidroponía: una solución de producción en Chihuahua, México. Boletín Informativo de la Red Hidroponía Nº 9. Lima, Perú.

Rotar, P. 2006. Hydroponic techniques sprout, healthy, inexpensive fodder (en línea). Consultado el 19 may. 2006. Disponible en: http://www.isar.org/pubs/ST/ hydroponics47.html
SAS (Statistical Analysis System). 1999. SAS User's guide: Statistics (Version 8.2 Ed). SAS Institute Inc. Cary, NC. 373 p.

Sneath, R; Mcintosh, F. 2003. Review of hydroponic fodder production for beef cattle. On farm. Meat \& Livestock Australia Limited. Australia. 54 p.

Sosa de Pro, E. 1979. Manual de procedimientos analíticos para alimentos de consumo animal. Chapingo, México. 115 p.

Tarrillo, H. 2007. Forraje verde hidropónico, forraje de alta calidad, para la alimentación animal (en línea). Arequipa, Perú. Consultado el 15 jun. 2007. Disponible en: http:// www.ofertasagricolas.cl/artículos/print.php?id=88

Taiz, L; Zerge, E. 2003. Fisiología vegetal. $3^{\circ}$ edición. Editorial Artemed, Porto Alegre Brasil. 720 p.

Valdivia, E. 1997. Producción de forraje verde hidropónico. Conferencia Internacional de Hidroponía Comercial. Lima, Perú. p. 59.

Van Soest, P. 1994. Nutritional ecology of the ruminant. New York. Cornell University Press. 2 ed. 463 p. 